\title{
Comparison of the orientation of small-scale electron density irregularities and $F$ region plasma flow direction
}

\author{
E. D. Tereshchenko ${ }^{1}$, B. Z. Khudukon ${ }^{1}$, M. O. Kozlova ${ }^{1}$, O. V. Evstafiev ${ }^{1}$, \\ T. Nygrén ${ }^{2}$, M. T. Rietveld ${ }^{3}$, A. Brekke ${ }^{4}$ \\ ${ }^{1}$ Polar Geophysical Institute, 15 Khalturina, 183010 Murmansk, Russia \\ 2 Department of Physical Sciences, University of Oulu, P.O. Box 3000, FIN-90401 Oulu, Finland \\ ${ }^{3}$ EISCAT, N-9027 Ramfjordbotn, Norway; also at Max-Planck-Institut für Aeronomie, D-37191 Katlenburg-Lindau, Germany \\ ${ }^{4}$ Auroral Observatory, University of Tromsø, N-9037 Tromsø, Norway
}

Received: 5 May 1999 / Revised: 13 March 2000 / Accepted: 17 March 2000

\begin{abstract}
Results are shown from an experimental campaign where satellite scintillation was observed at three sites at high latitudes and, simultaneously, the F region plasma flow was measured by the nearby EISCAT incoherent scatter radar. The anisotropy parameters of field-aligned irregularities are determined from amplitude scintillation using a method based on the variance of the relative logarithmic amplitude. The orientation of the anisotropy in a plane perpendicular to the geomagnetic field is compared with the direction of $F$ region plasma flow. The results indicate that in most cases a good agreement between the two directions is obtained.
\end{abstract}

Key words: Ionosphere (auroral ionosphere; ionospheric irregularities)

\section{Introduction}

Investigation of the anisotropy of the F layer irregularities is of great interest in ionospheric physics. It is well known that the irregularities are elongated in the direction of the local geomagnetic field but, especially at high latitudes, they are also found to be anisotropic in the plane perpendicular to the magnetic field vector. Irregularities which are statistically symmetric around the geomagnetic field are often called 'rods' and those which are anisotropic also in the perpendicular plane are referred to as 'sheets' or 'wings' (e.g. Livingston et al., 1982). The difference between sheets and wings is that the former ones have similar field-aligned and fieldperpendicular elongations ( $\alpha$ and $\beta$, respectively), so that $\alpha=\beta>1$, whereas wings are elongated more in the

Correspondence to: T. Nygrén e-mail: tuomo.nygren@oulu.fi direction of the geomagnetic field than in the perpendicular plane, i.e. $\alpha>\beta>1$.

The anisotropy is usually investigated by correlation analysis of radio signals from satellites, observed by spaced receivers on the ground, or by comparing model curves of the $S 4$ amplitude scintillation index to experimental ones. In most studies the irregularities seem to be elongated in the magnetic east-west direction (Martin and Aarons, 1977; Fremouw et al., 1977; Rino et al., 1978; Rino and Livingston, 1982), but sometimes the orientation is found to be rather in the north-south direction (Moorcroft and Arima, 1972). Rod-like irregularities have also been reported (Pryse et al., 1991). Livingston et al. (1982) observe all three types of irregularities and attach the various types to different parts of the convective plasma flow pattern.

The previous works do not actually determine the direction of the field-perpendicular anisotropy but rather give some rough estimates on its orientation. Recently, Tereshchenko et al. (1999) presented a new way of determining the anisotropy parameters of highlatitude $\mathrm{F}$ layer irregularities. This method works on data from a single site and it also gives the direction of the perpendicular anisotropy. It makes use of amplitude scintillation and therefore observes small-scale irregularities with sizes of the order of the Fresnel radius (Yeh and Liu, 1982). In the present case the Fresnel radius is about $600-700 \mathrm{~m}$.

It is believed that electric fields in the high-latitude $\mathrm{F}$ region play an important role in the generation of smallscale irregularities. Therefore one might expect a close correlation between the electric field direction and the orientation of the field-perpendicular anisotropy. In this study a modified version of the method by Tereshchenko et al. (1999) is presented and applied to data collected at three sites in Northern Scandinavia in November 1977. The experiments were made close to the EISCAT radar, which was used for determining the $F$ region plasma flow. The two data sets are used for comparing the plasma flow direction and the orientation of the perpendicular anisotropy. 


\section{Scintillation method}

In the previous works by Tereshchenko et al. $(1998,1999)$ the statistical parameter used in fitting the model to observations was the mean logarithmic relative amplitude. It was also shown that this parameter has a close relationship to the variance of the logarithmic relative amplitude. Since the variance actually works well to describe the level of amplitude scintillation, the method has been revised to use this parameter instead of the mean logarithmic relative amplitude. In the following the modified version is briefly outlined and some parts of the theory not shown in previous studies are also presented.

The use of the scintillation method implies that the spectrum of the irregularities obeys the power law. This is not a limitation, since the validity of the assumption has been shown e.g. by Secan et al. (1997), Aarons (1982) and Tereshchenko et al. (1998, 1999). It is also assumed that the irregularities make a statistically homogeneous layer spreading horizontally within a wide enough horizontal region. When a radio signal from the satellite is scattered by the layer of irregularities, a maximal scintillation is encountered at some specific direction of the ray from the radio source to the receiver. This is a result of a geometric effect defined by the ray direction, the geomagnetic field direction and the anisotropy parameters of the irregularities. Using the known geomagnetic field and the direction where the maximal scintillation is observed, one can determine the parameters of three-dimensional anisotropy from certain statistical parameters of the amplitude of the scattered signal.

In terms of the Rytov method and using the notations by Tereshchenko et al. (1999), the variance of the logarithmic relative amplitude $\chi=\ln A / A_{0}$ is

$\sigma_{\chi}^{2}=\frac{\lambda^{2} r_{e}^{2}}{4 \pi^{2}} \int_{z_{l}}^{z_{u}} \mathrm{~d} z \iint_{-\infty}^{\infty} \mathrm{d} \boldsymbol{\kappa}_{x y} \Phi\left(z \mathbf{e}_{\mathbf{z}}, \boldsymbol{\kappa}_{x y}\right) \sin ^{2} \frac{R_{F}^{2} \kappa_{x y}^{2}}{4 \pi}$.

Here $\lambda$ is the wave length, $r_{e}$ is the classical electron radius, the $z$ axis is oriented along the ray direction, $z_{l}$ and $z_{u}$ are the lower and upper boundary of the layer of irregularities, $R_{F}$ is the Fresnel radius, $\boldsymbol{\kappa}_{x y}$ is the component of the wave vector in a plane perpendicular to the $z$ axis and $\Phi$ is the spectral density of the fluctuations. The $y$ axis is taken to lie in the plane defined by the ray and the geomagnetic field vector, and the $x$ axis perpendicular to this plane. In the definition of the logarithmic relative amplitude, $A$ is the observed signal amplitude and $A_{0}$ its (unknown) value without irregularities.

The spectrum of the irregularities can be written as

$\Phi(\mathbf{r}, \mathbf{\kappa})=\sigma_{N}^{2}(\mathbf{r}) \Phi_{0}(\mathbf{r}, \mathbf{\kappa})$,

where $\Phi_{0}(\mathbf{r}, \mathbf{\kappa})$ is the normalized spectral density and $\sigma_{N}^{2}(\mathbf{r})$ is the variance of density fluctuations. With this notation Eq. (1) then gives

$\sigma_{\chi}^{2}=\frac{\lambda^{2} r_{e}^{2}}{4 \pi^{2}} \int_{z_{l}}^{z_{u}} \mathrm{~d} z \sigma_{N}^{2}(z) \iint_{-\infty}^{\infty} \mathrm{d} \boldsymbol{\kappa}_{x y} \Phi_{0}\left(z \mathbf{e}_{\mathbf{z}}, \boldsymbol{\kappa}_{x y}\right) \sin ^{2} \frac{R_{F}^{2} \kappa_{x y}^{2}}{4 \pi}$.
In this expression the double integral depends only on the shape of the spectrum and is independent of the variance of density fluctuations.

Next we assume that the normalized spectrum obeys the power law

$$
\begin{aligned}
\Phi_{0(p)}(\mathbf{\kappa})= & \frac{\alpha \beta L_{0}^{3} \Gamma(p / 2)}{2 \pi \Gamma(3 / 2) \Gamma[(p-3) / 2]} \\
& \times\left[1+\left(\frac{L_{0}}{2 \pi}\right)^{2}\left(\alpha^{2} \kappa_{\|}^{2}+\beta^{2} \kappa_{x \perp}^{2}+\kappa_{y \perp}^{2}\right)\right]^{-p / 2} .
\end{aligned}
$$

Here $\Gamma$ is the gamma function, $p$ is the power index, $L_{0}$ is the scale size of the irregularities, and $\kappa_{\|}, \kappa_{x \perp}$ and $\kappa_{y \perp}$ are the three components of the wave number vector $\boldsymbol{\kappa}$ in directions parallel and perpendicular to the geomagnetic field. The orientation of the $x_{\perp}$ and $y_{\perp}$ axes is chosen to make the field-perpendicular anisotropy aligned with the $x_{\perp}$ direction. With this spectral shape Eq. (3) gives

$$
\begin{aligned}
\sigma_{\chi}^{2}= & \frac{\lambda^{2} r_{e}^{2} \alpha \beta L_{0}^{3-p} \pi^{(p-1) / 2}}{\Gamma[(p-3) / 2] \sin (p \pi / 4)} \\
& \times \int_{z_{l}}^{z_{u}} \mathrm{~d} z \frac{\sigma_{N}^{2}(z)}{\sqrt{1+\gamma^{2}(z)}} R_{F}^{p-2}(z) F\left[1-\frac{p}{2}, \frac{1}{2}, 1, \frac{\gamma(z)}{1+\gamma(z)}\right] \\
& \times\left[c(z)-\sqrt{a^{2}(z)+b^{2}(z)}\right]^{-p / 2}
\end{aligned}
$$

where

$\gamma=\frac{2 \sqrt{a^{2}(z)+b^{2}(z)}}{c(z)-\sqrt{a^{2}(z)+b^{2}(z)}}$

and

$$
\begin{aligned}
& a=\frac{1}{2}\left[\left(\alpha^{2}-1\right) \sin ^{2} \Theta(z)\right. \\
& \left.+\left(\beta^{2}-1\right)\left(\sin ^{2} \Psi \cos ^{2} \Theta(z)-\cos ^{2} \Psi\right)\right] \\
& b=\left(\beta^{2}-1\right) \sin \Psi \cos \Psi \cos \Theta(z) \\
& c=1+\frac{1}{2}\left[\left(\alpha^{2}-1\right) \sin ^{2} \Theta(z)\right. \\
& \left.+\left(\beta^{2}-1\right)\left(\sin ^{2} \Psi \cos ^{2} \Theta(z)+\cos ^{2} \Psi\right)\right] .
\end{aligned}
$$

In these formulas $\Theta(z)$ is the angle between the geomagnetic field and the ray, $F$ is the hypergeometric function, and $\Psi$ is the angle between the $x$ and $x_{\perp}$ axes.

The hypergeometric function reaches its maximum value at $\Theta=0$, i.e. at the magnetic zenith. It decreases when moving away from this direction, and it affects the integral only within a narrow cone around the magnetic zenith. The slowly varying Fresnel radius can be easily calculated for each satellite trajectory and the angle $\Theta(z)$ is obtained from the magnetic field model. Therefore $\sigma_{\chi}^{2}$ can be calculated for given values of the anisotropy parameters $\alpha, \beta$ and $\Psi$ and a given model of $\sigma_{N}^{2}(z)$. The values of $\alpha, \beta$ and $\Psi$ can be determined by varying their values until the best fit to the experimental curve is obtained. 
In conclusion, the foregoing discussion shows that the variance of logarithmic relative amplitude contains information on the anisotropy of $\mathrm{F}$ region irregularities in three dimensions. However, the experimental determination of $\chi$ itself is not possible since $A_{0}$ is not known. Instead, we can estimate the quantity

$\chi_{e}=\ln \frac{A}{\langle A\rangle}$,

and therefore one should investigate how its variance $\sigma_{\chi_{e}}^{2}$ is related to $\sigma_{\gamma}^{2}$.

Starting from the result by Rytov et al. (1978)

$\langle A\rangle=A_{0} \exp \left(\langle\chi\rangle+\sigma_{\chi}^{2} / 2\right)$

and remembering that $\langle\chi\rangle=-\sigma_{\chi}^{2}$ (see Tereshchenko et al., 1999), we readily obtain

$\chi_{e}=\chi+\sigma_{\chi}^{2} / 2$.

Then obviously $\left\langle\chi_{e}\right\rangle=-\sigma_{\chi}^{2} / 2$ and

$$
\begin{aligned}
\sigma_{\chi_{e}}^{2} & =\left\langle\left(\chi_{e}-\left\langle\chi_{e}\right\rangle\right)^{2}\right\rangle=\left\langle\left(\chi+\sigma_{\chi}^{2}\right)^{2}\right\rangle \\
& =\left\langle(\chi-\langle\chi\rangle)^{2}\right\rangle=\sigma_{\chi}^{2}
\end{aligned}
$$

so that the variances of $\chi$ and $\chi_{e}$ are identical. This means that, if $\sigma_{\chi_{e}}^{2}$ is calculated from experimental estimates of $\chi_{e}$, the result can be directly compared to $\sigma_{\chi}^{2}$ calculated from Eq. (5).

When $\sigma_{\chi}^{2}$ is plotted as a function of the latitude of the satellite position (or the latitude of the point where the ray crosses a reference height within the layer), the resulting curve has a single maximum. The satellite path, the local magnetic field and the values of the anisotropy parameters define the shape and the position of the peak. Hence the anisotropy axial ratios $\alpha$ and $\beta$ and the orientation of the field-perpendicular anisotropy $\Psi$ can be determined from amplitude measurements at a single site by fitting a theoretical model to observations. Instead of $\Psi$, the orientation of the perpendicular anisotropy will be given below in terms of $\varphi$, which is the directional angle of the horizontal projection of perpendicular anisotropy axis, measured eastwards starting from geographic north.

\section{Experimental campaign}

In October 1997, a satellite tomography campaign was arranged in northern Scandinavia with a chain of five receivers at Oulu $\left(65.06^{\circ} \mathrm{N}, 25.47^{\circ} \mathrm{E}\right)$ in Finland, Esrange $\left(67.88^{\circ} \mathrm{N}, 21.06^{\circ} \mathrm{E}\right)$ in Sweden as well as Nordkjosbotn $\left(69.22^{\circ} \mathrm{N}, 19.54^{\circ} \mathrm{E}\right)$, Tromsø $\left(69.59^{\circ} \mathrm{N}, 19.22^{\circ} \mathrm{E}\right)$ and Kårvika $\left(69.87^{\circ} \mathrm{N}, 18.93^{\circ} \mathrm{E}\right)$ in Norway. Only amplitude data from the three northernmost sites is used in this work. Their corrected geomagnetic longitudes are between $103.56^{\circ}$ and $103.66^{\circ}$ so that they lie quite precisely on the same geomagnetic meridian.

The satellites used in the experiment fly at an altitude of about $1000 \mathrm{~km}$ approximately along a geomagnetic meridian during their southward passages, and the receivers carry out difference Doppler measurements at two frequencies (150 and $400 \mathrm{MHz}$ ). The receiving system is basically the same as that described by Chernyakov et al. (1993); it was only developed to allow, in addition, the measurement of the $150 \mathrm{MHz}$ signal amplitude for the purpose of scintillation research. The system of data collection was also completely revised.

The tristatic EISCAT UHF radar was operated in two different modes. One of them was plain CP-1-K, which is one of the standard modes designed for measuring both $\mathrm{D}, \mathrm{E}$ and $\mathrm{F}$ regions. It applies short and long pulses as well as alternating codes. The Tromsø beam direction is field-aligned, and the tristatic volume for measuring the $F$ region drift lies at $250 \mathrm{~km}$ altitude. The second mode was otherwise similar to CP-1-K but the beam directions were controlled by an external FORTRAN programme simulating manual beam-positioning commands. Different beam swings were used from case to case and some variation of the altitude of the tristatic volume also took place. The purpose of this procedure was to make the radar beam move more or less in phase with the passing satellite.

The $\mathrm{F}$ region radar data are used here for measuring the plasma drift velocity. The quality of the radar data was not consistently good; quite often only data from two sites are available or the data are faulty so that incoherent scatter analysis completely fails. If only two velocity components are available, the plasma flow is assumed to be perpendicular to the geomagnetic field. When data from all three sites are complete, the total velocity vector is determined.

\section{Analysis of scintillation data}

The sampling frequency in the satellite receiver systems was $50 \mathrm{~Hz}$. The variance estimates were calculated from sequences of 500 successive data points in the amplitude time series (corresponding to 10-s intervals in time) and this data window was shifted in steps of 50 points (corresponding to $1 \mathrm{~s}$ in time). A linear fit was made to the data points within each 500-point interval and, for each sampling time, the fitted value $\langle A\rangle_{e}$ was taken as the experimental estimate of the mean value $\langle A\rangle$. Next the quantity $\ln \left(A /\langle A\rangle_{e}\right)$ was determined for each sampling time and its variance $\sigma_{\chi_{e}}^{2}=\left\langle\left(\ln \left(A /\langle A\rangle_{e}\right)-\right.\right.$ $\left.\left.\left\langle\ln \left(A /\langle A\rangle_{e}\right)\right\rangle\right)^{2}\right\rangle$ was calculated. This procedure gives a sliding estimate of $\sigma_{\chi_{e}}^{2}$ in steps of $1 \mathrm{~s}$, each value being obtained from a period of $10 \mathrm{~s}$. The results were plotted as a function of the corrected geomagnetic latitude of the point where the ray joining the satellite and the receiver crosses the centre of the model scintillating layer.

In order to have a correct power index to be used in Eq. (5), the power law spectral shape was fitted to the high-frequency part of the spectral density of the amplitude data. The fitting was made in the vicinity of the peaks in the variance curves, separately to data from the three sites, and the mean value of the power index was then used in calculating the theoretical $\sigma_{\gamma}^{2}$.

The anisotropy parameters were determined by fitting the theoretical curve of $\sigma_{\chi}^{2}$, calculated from 
Eq. (5), to the experimental one. The fitting procedure is similar to that explained by Tereshchenko et al. (1999). The applied model of density fluctuations is a statistically homogeneous sheet (constant $\alpha, \beta, \Psi$ and $\sigma_{N}^{2}$ ) with a thickness of $100 \mathrm{~km}$, centred at the height of the tristatic volume of the incoherent scatter radar.

The position of the maximum in the $\sigma_{\chi}^{2}$ curve depends mainly on $\Psi$ and the width of the peak depends mainly on $\alpha$ and $\beta$. Estimates of the error limits were obtained for these parameters by investigating their variations which would keep the maximum point in latitude within $0.1^{\circ}$ from the best value without noticeable changes of the peak shape.

This method works well in determining the anisotropy parameters, if the satellite does not pass too close to or too far from the local magnetic zenith. The critical parameter here is $\Theta_{\min }(z)$, which should optimally lie within the range $3^{\circ}-15^{\circ}$. If $\Theta_{\min }(z)$ is too close to zero, $\sigma_{\chi}^{2}$ is insensitive to the orientation of perpendicular anisotropy. If $\Theta_{\min }(z)$ is large, i.e. the satellite flies too far from the receiver site, the spectrum and anisotropy of the irregularities hardly remain constant at large distances. Horizontal variations in these two factors may create multiple maxima in the variance curves which cannot easily be distinguished from the peak due to the straightforward geometrical effect. In order to satisfy the demand of a small value of $\Theta_{\min }(z)$, only satellite passages with highest elevations exceeding $80^{\circ}$ were used in the analysis.

Finally one should point out that, although the peak in the variance curve corresponds to a specific ray direction, the values of the anisotropy parameters do not come from a single point in the $\mathrm{F}$ layer but rather they are descriptive of a region of a wider horizontal extent. The use of a statistically homogeneous infinite model layer means in practice that the method assumes the anisotropy parameters to remain constant within such a region. Its size, however, cannot be determined from data obtained at a single site. When the same parameters are found to apply to data from several sites, it will be possible to get information on the horizontal scale where the layer remains statistically homogeneous.

\section{Results}

Measurements of signal amplitude were made over almost 10 days, mostly in parallel with EISCAT observations. In spite of this, only nine sets of simultaneous scintillation and radar data sets could be selected for the analysis. This was caused by the fact that data gaps were quite common in the radar beam swing experiments and, furthermore, a low signal-to-noise ratio often reduced the reliability of the result. Also, in order to make the comparison meaningful, the scintillation and radar observations had to come from regions which are close enough to each other. With the present radar experiments, this implies satellite paths passing close to the local zenith, but this is also a demand posed by the method of scintillation analysis.
In some cases the spatial distances of the EISCAT and scintillation observations were not small or no radar measurement exactly simultaneous with the scintillation observation was available. Even then a comparison was sometimes possible using spatial or temporal trends of the electric field behaviour. Of course, the uncertainty of the comparison increases with separation in space or time. In these cases special care was taken in investigating the spatial and temporal structures of the electric field.

Three examples of different types will be shown. They all come from a period of quiet magnetic conditions and therefore the results are not necessarily representative of active periods like magnetic storms.

The satellite passage starting at about 2224 UT on 9 November, 1997, will be first investigated. Amplitudes from the separate amplitude channel and phases calculated from the quadrature components are plotted in Fig. 1. Enhanced amplitude scintillation is clearly visible in the centre parts of the left hand panels. Scintillation can also be seen in the phase, superimposed on smooth phase curves.

The variances of the logarithmic relative amplitude were calculated from the amplitude data in Fig. 1. The results were plotted in Fig. 2 using heavy lines. At each site a clear peak is created at the latitudes of the maximum scintillation level. At Kårvika and Tromsø the peaks are rather symmetric but at Nordkjosbotn a clearly asymmetric peak is encountered. In addition to the main peaks, smaller maxima are observed at other latitudes.

The spectra calculated from the amplitude data indicate that a power index $p=11 / 3$ can be used throughout in the fluctuation spectrum. A $100-\mathrm{km}$ thick layer with a constant variance of electron density fluctuations, centred around $255 \mathrm{~km}$ altitude, was used as a scintillation model. The positions of the peaks in all three curves are properly reproduced by the same model with $\alpha=28, \beta=5$ and $\varphi=76^{\circ}$. The best fit to Troms $\varnothing$ data is plotted as a thin line in the middle panel of Fig. 2. The thin lines in the top and bottom panels indicate the variances at Kårvika and Nordkjosbotn calculated with the same model parameters.

Figure 2 indicates that the anisotropy parameters given by the best fit to Tromsø data do indeed produce peaks with proper widths at correct latitudes at all three sites. This means that the same anisotropy parameters apply within the horizontal range covered by the three observations. The fact that the peak heights do not match can be explained by assuming a horizontal variation of $\sigma_{N}^{2}$. The same reason may also explain the asymmetric shape of the peak observed at Nordkjosbotn.

The directions of the orientation angles with their error limits are plotted as a function of the corrected geomagnetic latitude of the $\mathrm{F}$ region observation point in Fig. 3. In addition, the direction of the $F$ region plasma flow and its errors, calculated from the EISCAT observations in the period 2233:10-2235:00 UT, is plotted. This time interval closely covers the observation times of the scintillation peaks. The direction of local 

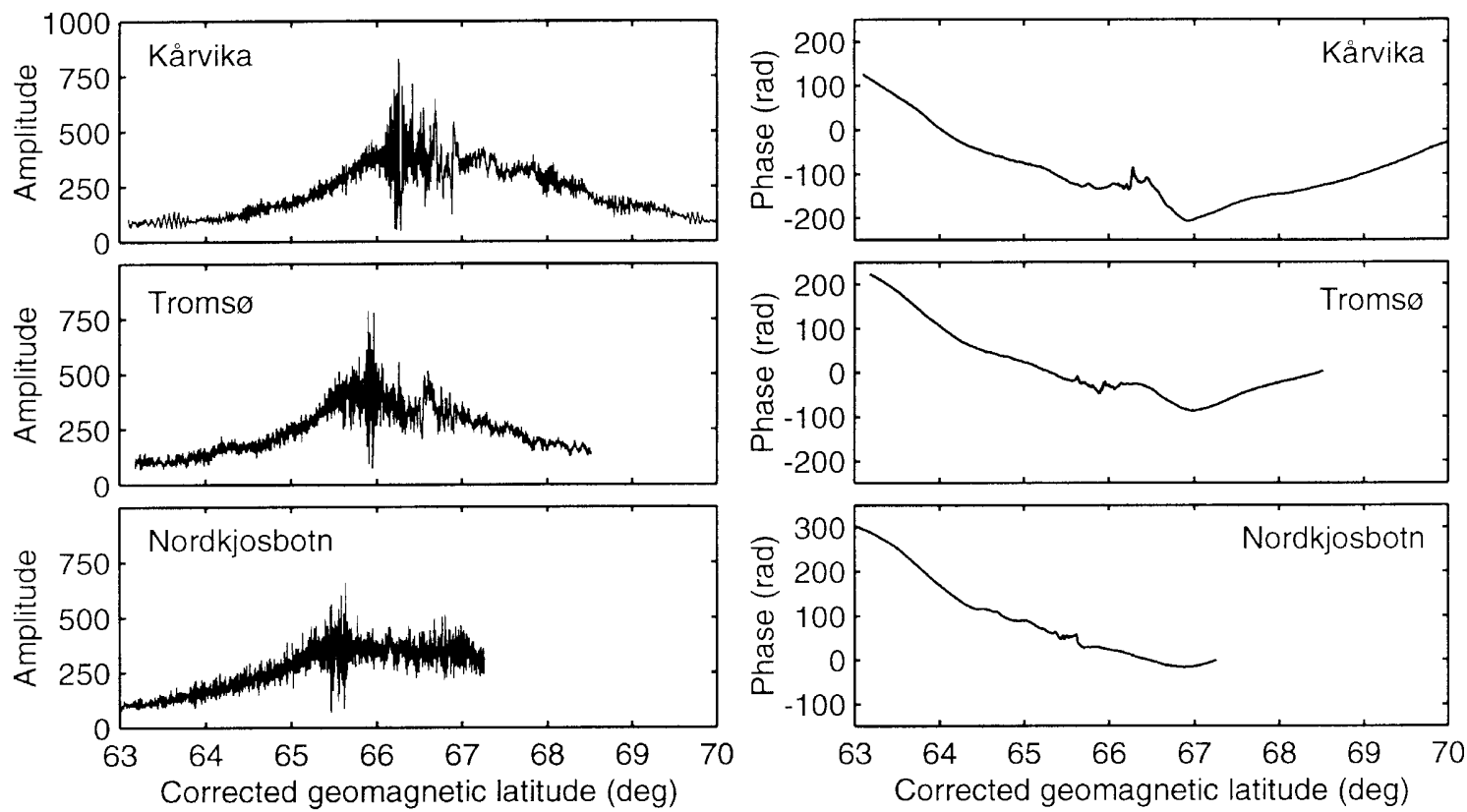

Fig. 1. Amplitudes and phases at Kårvika, Tromsø and Nordkjosbotn from a satellite passage starting at about 2224 UT on 9 November, 1997 (only pieces of the data are shown). The horizontal axis is the corrected geomagnetic latitude of the point where the ray crosses the reference height

geomagnetic east at each observation point is also shown in Fig. 3.

The maximum points in the variance curves all lie within a range of about $0.7^{\circ}$ in latitude. Hence the anisotropy parameters of the irregularities seem to stay fairly constant at least within this latitudinal range. Also, the distance between the EISCAT measurement point and the maximum point at Tromsø is only about $15 \mathrm{~km}$ so that these two measurements come nearly from the same location. It is found that, within the error limits, the plasma flows in the direction of the perpen- dicular anisotropy determined from the Tromsø observation, i.e. the electric field is approximately orthogonal to the axis of field-perpendicular anisotropy. One should notice that the direction of observed perpendicular anisotropy departs from the local magnetic east by $15-20^{\circ}$

The direction of the horizontal component of the $\mathrm{F}$ region plasma velocity around the time of the observation in Fig. 3 is plotted in Fig. 4. The field first rotates continuously towards north via east, but then turns back to the original direction again. The direction closest to

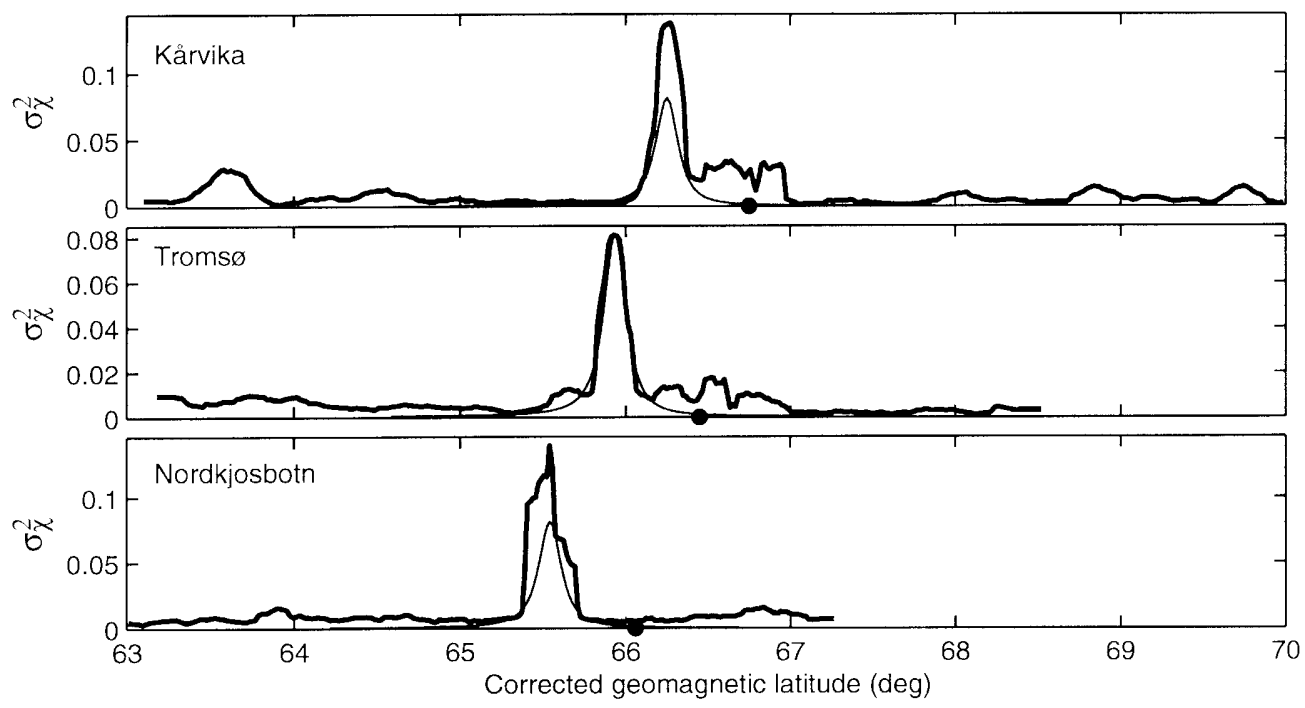

Fig. 2. The variance of the logarithmic relative amplitude calculated from the amplitude data in Fig. 1 (bold line). The receiver sites are indicated by large dots. The scintillation model is a layer with constant variance of electron density fluctuations and a thickness of $100 \mathrm{~km}$, centred at a reference height of $255 \mathrm{~km}$. The thin line in the middle panel shows the best fit of the model to Tromsø data and in the top and bottom panel the variances calculated with the same model parameters at Kårvika and Nordkjosbotn, respectively. The horizontal axis is the corrected geomagnetic latitude of the point where the ray crosses the reference height 
9 Nov 1997, 2233-2235 UT

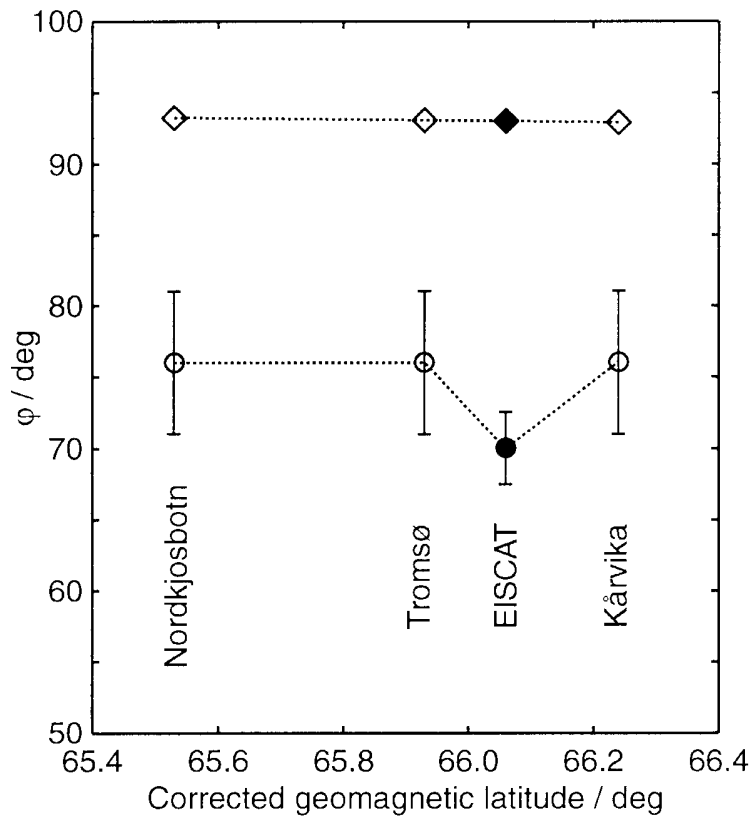

Fig. 3. The directional angles $\varphi$ of the fitted model in Fig. 2 at the three sites (open dots) with error bars as a function of the corrected geomagnetic latitude of the observation points. The directional angle of the F region ion drift measured by EISCAT is shown by a solid dot. The direction of local magnetic east at respective points is indicated by diamonds

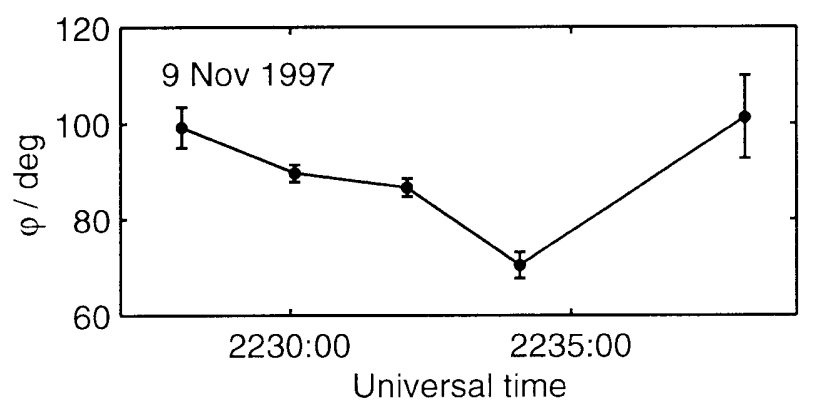

Fig. 4. Time variation of the directional angle of the plasma velocity during the satellite passage starting at 2224 UT on 9 November, 1997, as observed by the EISCAT radar

north corresponds to the situation in Fig. 3. These measurements come mainly from a southward beam swing, but the two last points are from the same beam direction (there is one beam direction in between with faulty data which could not be analyzed). Therefore the observed variations are temporal, although the possibility of horizontal variations cannot be excluded. The systematic change with small error limits indicate a smooth continuous turning of the flow direction. From this time-dependent period we have a single scintillation observation shown in Fig. 3 which agrees well with the plasma flow direction just at the time of the scintillation observation.

Results of a similar analysis from a satellite passage starting at 0154 UT on 10 November, 1997 are shown in Fig. 5. In this case scintillation was observed at all three

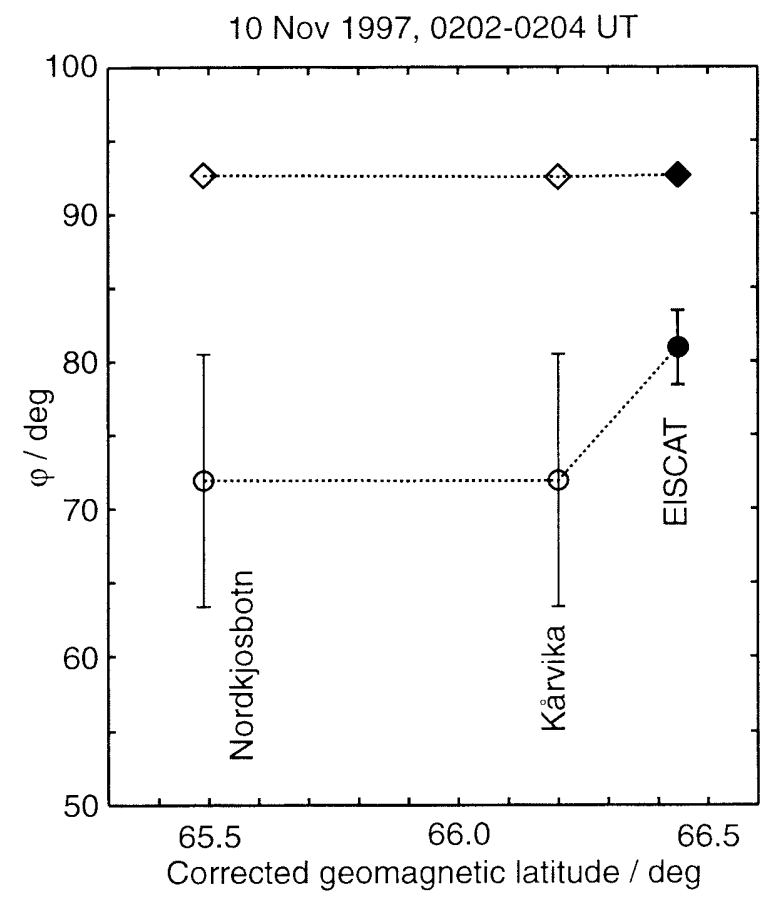

Fig. 5. Same as Fig. 3

sites during the whole period of the satellite passage. However, a clear peak was seen at Kårvika and two distinct peaks at Nordkjosbotn, but no isolated maximum was seen at Tromsø. The same model could be applied in fitting the Kårvika data and one of the peaks at Nordkjosbotn. The height of the model centre was $259 \mathrm{~km}$ and the fitted anisotropy parameters were $\alpha=25, \beta=3.5$ and $\varphi=72^{\circ}$. The directional angle is plotted in Fig. 5 together with the plasma flow direction from the period 00202:150204:00 UT. In this case the error limits of the anisotropy direction are larger than in Fig. 3. This is because the satellite passes the receivers quite close to the local magnetic zenith, the minimum zenith angles varying within a range of $0.2-2^{\circ}$. In such a case the perpendicular anisotropy has only a small effect on $\sigma_{\gamma}^{2}$, which reduces the accuracy of the direction measurement (see Tereshchenko et al., 1999).

In this case the distances between the EISCAT measurement volume and the points, where the variance reaches its maximum at Nordkjosbotn and Kårvika, are $30-100 \mathrm{~km}$. One might suspect the ionospheric parameters could change within this distance. Nevertheless, Fig. 5 shows again that the directions of plasma flow and perpendicular anisotropy agree within the error limits. The fact that the irregularities are not observed at Tromsø could be explained in terms of horizontal variation of the parameters of the irregularities.

Figure 6 shows the direction of the horizontal component of the $F$ region plasma velocity during the satellite passage of the observation in Fig. 5. The radar beam was fixed during this interval so that all measurements come from the same point. It is seen that the flow direction was first rather stable before about 0156 UT, but then somewhere between 0156 UT and 0200 UT a change took place, after which the original direction was 


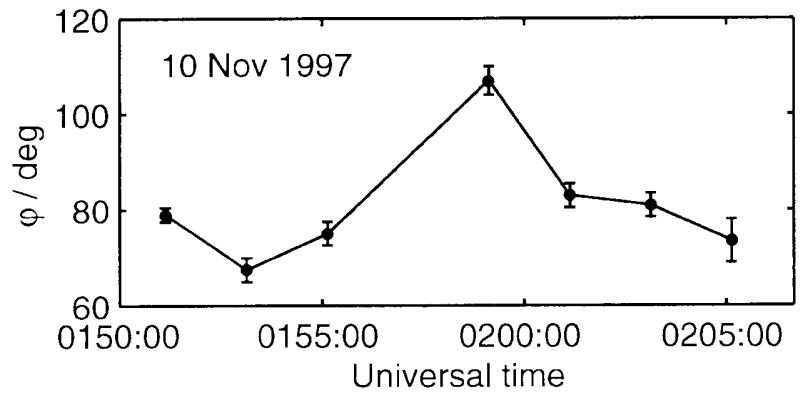

Fig. 6. Same as Fig. 4 during the satellite passage starting at 0154 UT on 10 November, 1997

nearly restored. The maxima in $\sigma_{\gamma}^{2}$ were observed at Nordkojsbotn and Kårvika right after the disturbance. The temporal variations in the plasma flow may have caused local changes in density fluctuations which made them unobservable at Tromsø.

A third example, from the satellite passage starting at 1231 UT on 11 November, 1997, is shown in Fig. 7. The amplitude data give clear peaks in the variance curves at all three sites, but this example differs from the previous ones in the sense that the same anisotropy parameters do not apply to each data set. Fitting the model gives $\alpha=30, \beta=5$ and $\varphi=131^{\circ}$ at Kårvika, $\alpha=28, \beta=4$ and $\varphi=91^{\circ}$ at Tromsø, and $\alpha=23, \beta=3.5$ and $\varphi=79^{\circ}$ at Nordkjosbotn. This happens although the distances of the maximum points of the variance curves are only $14-42 \mathrm{~km}$. Hence the properties of the irregularity layer must vary considerably within a short distance. In particular, there is a great variation in the orientation of the perpendicular anisotropy. The fact that the EISCAT

11 Nov 1997 1239-1240 UT

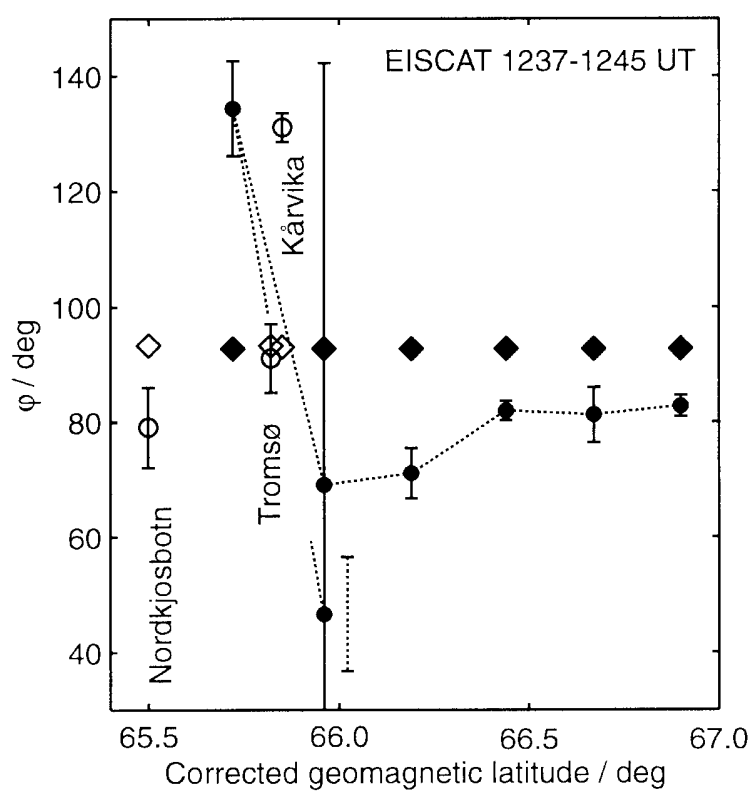

Fig. 7. Same as Fig. 3, except that for the EISCAT measurement, $\varphi$ indicates the direction opposite to the plasma velocity. Radar observations from six positions of a southward swing in the meridional plane and the next point in the following northward swing are shown heater was on during this observation may have affected the spatial variation of the anisotropy parameters, although this cannot be attested for certainty.

During this observation the radar beam was swung in a geographic meridional plane at $19.21^{\circ} \mathrm{E}$. The plasma flow directions shown in Fig. 7 come from a simultaneous southward meridional scan (since the flow vector has a westward component here, it is more convenient to define $\varphi$ to indicate the direction opposite to the plasma flow). Out of these points, the radar observation at a geomagnetic latitude $66.44^{\circ}(1238: 55-1241: 15$ UT) is simultaneous with the scintillation measurement. In addition, the next point from the following northward swing is also portrayed (with dashed error bar aside).

We notice that the flow direction in the southernmost point at a geomagnetic latitude $65.72^{\circ}$ strongly departs from the others. Also, the error limits in the neighbouring point at a geomagnetic latitude $65.96^{\circ}$ are very great in the southward scan, probably indicating that this observation point met a moving boundary of two different flow regions during the measurement at 1242:50-1243:15 UT. Much smaller error limits are encountered at the same point in the next northward scan. In general, however, the characteristics of the latitude profile remain the same in three subsequent scans indicating some stationarity in the flow pattern.

Horizontal variation in the flow pattern is obviously considerable in this example. Hence, since no radar measurement is available from the observation latitude of the Nordkjosbotn receiver, the anisotropy and flow directions cannot be compared. The Tromsø and Kårvika observations come from nearly the same geomagnetic latitudes $\left(65.82^{\circ}\right.$ and $65.85^{\circ}$, respectively) but from somewhat different geomagnetic longitudes (104.34 ${ }^{\circ}$ and $104.13^{\circ}$, respectively). The Kårvika observation comes from a position closer to the southernmost radar observation and therefore one might expect a closer agreement between the orientation of the anisotropy at Kårvika and the flow direction at the southernmost beam position. Indeed, the flow direction in the southernmost point agrees quite well with the Kårvika observation. This is not a definite proof, however, since the radar measurement comes from a more southern point and from 4 min later in time than the scintillation observation. Nothing very specific can be said about the Tromsø observation, but at least it is not in a clear disagreement with the plasma flow pattern.

Further evidence on the the different physical surroundings of the three scintillation measurements is given by Fig. 8, which portrays the phases (i.e. the total electron contents) observed at the three sites. In each case the phase curve reveals a minimum and a local maximum to the north of it. These are obviously associated with pronounced large-scale density gradients in the F layer and the scintillation observations made at the three sites come from regions of different density gradients. Hence, both the $\mathrm{F}$ region plasma flow and electron density behave quite differently at the three points of maximum variance and, consequently, it is understandable that the anisotropy parameters are also quite different. 


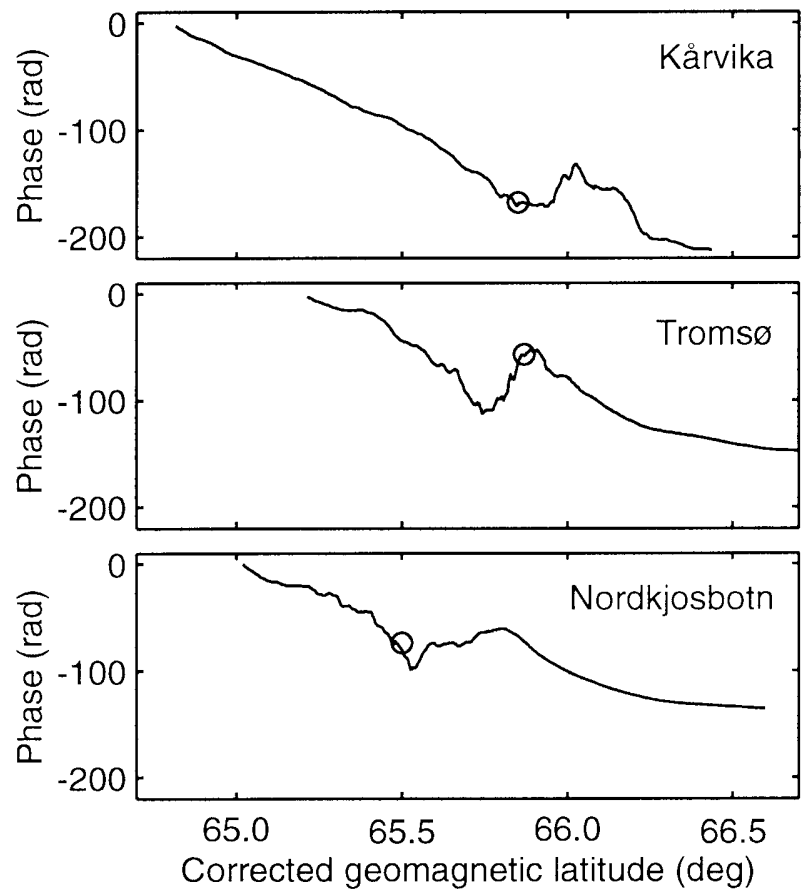

Fig. 8. Phases at Kårvika, Tromsø and Nordkjosbotn from a satellite passage starting at 1231 UT on 11 November, 1997. The latitudes of the scintillation observations are shown by circles

In these three examples it was found that, within the error limits and at least at one of the receiver sites, the perpendicular anisotropy is aligned in the direction of the plasma flow in the ionospheric $\mathrm{F}$ region. This also applies to the other cases investigated. In some of them, the radar observation was quite inaccurate and in others no simultaneous radar measurements were available. However, when the closest plasma flow observation was used in the latter ones, an agreement was again achieved. Hence no cases were found which would directly contradict the statement at the start of the paragraph.

\section{Discussion}

The physical results of the present work can be summarized as follows: in nearly all cases where the comparison of the anisotropy and the $\mathrm{F}$ region plasma flow was possible, the perpendicular anisotropy was oriented in the flow direction. This strongly suggests that the instability creating the fluctuations is driven by the electric field. The orientation is not necessarily along the geomagnetic east-west direction, but deviations of several tens of degrees do occur.

In previous works somewhat contradictory results on the orientation of perpendicular anisotropy have been obtained. The most common observation has been more or less east-west orientation (e.g. Martin and Aarons, 1977; Rino and Livingston, 1982) but occasionally north-south orientations have also been found (e.g. Moorcroft and Arima, 1972). The different types of irregularities like rods, sheets and wings are put in the common framework by Livingston et al. (1982), who connect them to different parts of the ionospheric plasma convection pattern.

All the older results have been obtained by methods which only give rough estimates on the orientation (north-south or east-west) or, alternatively, find the irregularities to be rod-like. The method presented originally by Tereshchenko et al. (1999) and used in a somewhat modified form here is essentially different: a specific direction with error limits is actually obtained as a result of fitting a model to observations. The other anisotropy parameters are simultaneously determined in the same fitting process. For the first time, these results allow the comparison of the directions of plasma flow and perpendicular anisotropy. One should also notice that this study deals with small-scale irregularities with sub-kilometre sizes, whereas irregularities with larger scale sizes are investigated in most of the previous papers.

The data set collected in the present campaign is too small to allow any statistical studies to be made and perhaps even too small to give a representative set of examples. Therefore more measurements should be made in the future. The number of examples for radar comparison was reduced by the quality of the radar data and occasional failures in the synchronization of the radar beams. These points can perhaps be improved in the future. Since the observation time of the EISCAT radars is necessarily limited, one should consider the use of coherent radars like STARE and CUTLASS, which are capable of mapping the ionospheric electric field within wide regions and could thus give data for comparing the orientation of anisotropy and the direction of plasma convection. An interesting possibility would be to use the dynasonde at Tromsø, which is also capable of giving plasma drift estimates (Sedgemore et al., 1998).

Acknowledgements. The authors are grateful to T.L. Hansen and A. Mortensen for their help in arranging the Kårvika receiver site as well as to A.P. van Eyken for the antenna scanning software and to R. Kuula for making the analysis of the incoherent scatter data. This work was supported by the Academy of Finland and by the Russian Foundation of Fundamental Research grant 1 98-0565049. The EISCAT measurements were made with special programme time granted to users outside the Association. EISCAT is an International Association supported by Finland (SA), France (CNRS), the Federal Republic of Germany (MPG), Japan (NIPR), Norway (NFR), Sweden (NFR) and the United Kingdom (PPARC).

The Editor in chief thanks J. Aarons and E. Pryse for their help in evaluating this paper.

\section{References}

Aarons, J., Global morphology of ionospheric scintillations, Proc. IEEE, 70, 360-378, 1982.

Chernyakov, S. M., E. D. Tereshchenko, B. E. Brunelli, and T. Nygrén, Comparison of ionospheric total electron content measured using the difference Doppler and incoherent scatter methods, Ann. Geophysicae, 11, 10-16, 1993.

Fremouw, E. J., C. L. Rino, R. C. Livingston, and M. C. Cousins, A persistent subauroral scintillation enhancement observed in Alaska, Geophys. Res. Lett., 4, 539-542, 1977. 
Livingston, R. C., C. L. Rino, J. Owen, and R. T. Tsunoda, The anisotropy of high-latitude nighttime $\mathrm{F}$ region irregularities, J. Geophys. Res., 87, 10 519-10 526, 1982.

Martin, E., and J. Aarons, F layer scintillations and the aurora, J. Geophys. Res., 82, 2717-2722, 1977.

Moorcroft, D. R., and K. S. Arima, The shape of the F-region irregularities which produce satellite scintillations - evidence for axial asymmetry, J. Atmos. Terr. Phys., 34, 437-450, 1972.

Pryse, S. E., L. Kersely, and C. D. Russell, Scintillation near the F layer trough over northern Europe, Radio Sci., 26, 1105-1114, 1991.

Rino, C. L., and R. C. Livingston, On the analysis and interpretation of spaced-receiver measurements of transionospheric radio waves, Radio Sci., 17, 845-854, 1982.

Rino, C. L., R. C. Livingston, and S. J. Matthews, Evidence for sheet-like auroral ionospheric irregularities, Geophys. Res. Lett., 5, 1039-1042, 1978.

Rytov, S. M., Y. A. Kratsov, and V. E. Tatarsky, Introduction to statistical radio-physics. Part II. Random processes. Nauka, Moscow, 463 p., 1978 (in Russian).
Secan, J. A., R. M. Bussey, E. J. Fremouw, and S. Basu, Highlatitude upgrade to the wide band ionospheric scintillation model, Radio Sci., 32, 1567-1574, 1997.

Sedgemore, K. J. F., J. W. Wright, P. J. S. Williams, G. O. L. Jones, and M. T. Rietveld, Plasma drift estimates from the Dynasonde: Comparison with EISCAT measurements, Ann. Geophysicae, 16, 1138-1143, 1998.

Tereshchenko, E. D., B. Z. Khudukon, M. T. Rietveld, and A. Brekke, Spatial structure of auroral day-time ionospheric electron density irregularities generated by powerful HF-wave, Ann. Geophysicae, 16, 812-820, 1998.

Tereshchenko, E. D., B. Z. Khudukon, M. O. Kozlova, and T. Nygrén, Anisotropy of ionospheric irregularities determined from the amplitude of satellite signals at a single receiver, Ann. Geophysicae, 17, 508-518, 1999.

Yeh, K. C., and C.-H. Liu, Radio wave scintillations in the ionosphere, Proc. IEEE, 70, 324-360, 1982. 